\title{
Research in Layout of Pavement Markings in Freeway Merging Area
}

\author{
Junchen Dai ${ }^{1, a}$, Qiaojun Xiang ${ }^{1, b}$ \\ ${ }^{1}$ School of Transportation, Southeast University, Nanjing 210096, People's Republic of China \\ adjc_xy@qq.com, bxqj@seu.edu.cn
}

Keywords: freeway, pavement markings, gap acceptance theory, merging area, acceleration lane Abstract. Currently, Chinese standards on pavement markings are unable to avoid the situation that the ramp vehicles in low speed merge to mainline prematurely without enough acceleration. The large speed difference could cause serious traffic accidents. When compared the Chinese standards with MUTCD in American, Chinese standards lack the provision of solid white line installed from the theoretical gore and extended downstream in freeway merging area as to prohibit the early merging maneuver. Therefore, this paper analyzed the characteristics of traffic stream in merging area and principle of designing acceleration lane length in China, proposed an algorithm for designing length of the solid white line with gap acceptance theory, and a demonstrating example was given.

\section{Introduction}

In freeway merging area, merging vehicles from entrance ramp often disturb the steady operation of traffic stream in mainline. In China, because of the short of effective measures on traffic control, vehicles tend to merge prematurely without enough acceleration. The large speed difference between merging vehicles and vehicles in mainline could cause serious traffic accidents. Therefore, research in reasonable geometry design of freeway and traffic control is of great importance. Considering the practicability, pavement markings are practical and effective traffic control devices. However, When compared the Chinese standards on pavement markings with standards of western developed countries, it could be found that Chinese standard Road Traffic Signs and Markings (GB5768-2009) ${ }^{[1]}$ and Specification for Layout of Highway Traffic Signs and Markings (JTGD82-2009) ${ }^{[2]}$ lacks the provision of solid white line installed from the theoretical gore and extended downstream on parallel speed-change lane (in order to facilitate the narrative, this paper call the markings on acceleration lane prohibit merging line) as contrasted with Manual on Uniform Traffic Control Devices (MUTCD-2009) ${ }^{[3]}$ in USA, and little research has been done in China. As noted earlier, for the reason that parallel speed-change lane is adjacent to mainline and no effective traffic control devices is installed, the premature merging or late diverging increased dangers in vehicles operation. The comparison is shown in Fig. 1.
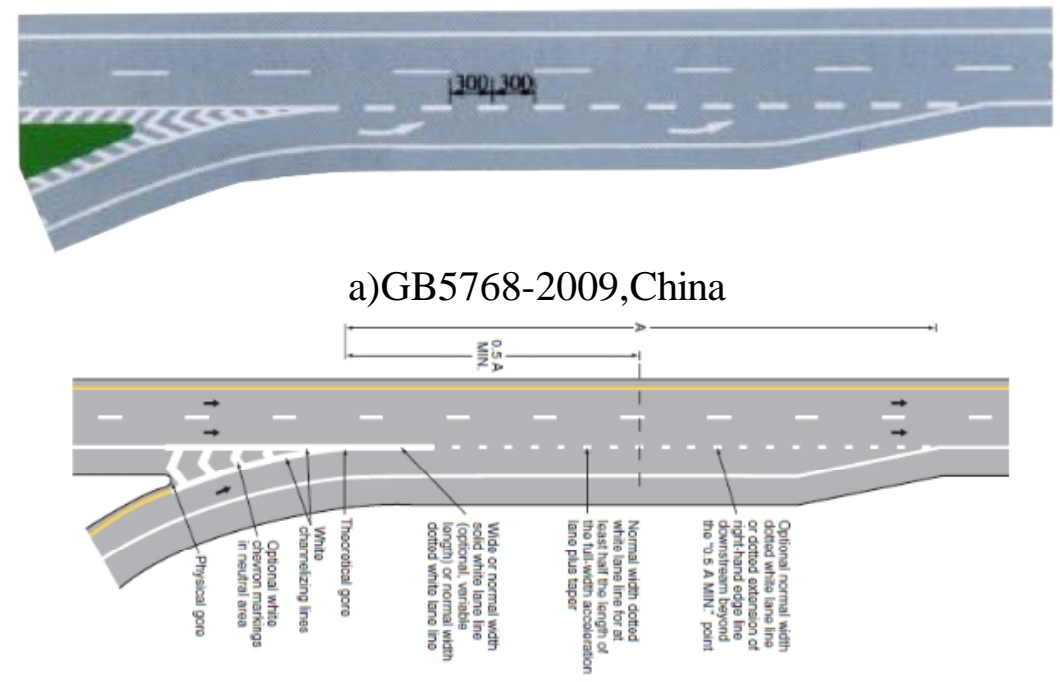

b) MUTCD-2009, American

Fig. 1 comparison in standards between China and American 
Design Specification for Highway Alignment (JTG- D20-2006) ${ }^{[4]}$ states that acceleration lanes with single lane should be designed as parallel mode, while acceleration lanes with double lanes and deceleration lanes should be designed as tapered mode, and for consideration of lane balance , acceleration lane with double lanes should use auxiliary lane, as shown in Fig.2, of which a) is tapered deceleration lane with single lane, b) is parallel acceleration lane with single lane, c) is tapered deceleration lane with double lanes, $\mathrm{d}$ ) is tapered acceleration lane with double lanes and an auxiliary lane. Generally speaking, because tapered speed-change lane is not adjacent to the mainline, if the length of speed-change lane is reasonable, the vehicles have already enough speed change when merging or diverging, and pavement markings shall not occupy the width of a lane, the problem mentioned above would not existed or could not solved by the pavement markings. However, to tapered acceleration lane with double lanes and auxiliary lane, for ramp vehicles could accelerate on auxiliary lane, and ramp vehicles on the inner lane tend to move to the outside lane to wait merging opportunity ${ }^{[5]}$, the demand for prohibit merging line is still existed. This paper analyzed the characteristics of traffic stream in merging area and principle of designing acceleration lane length, and study the layout of prohibit merging line.

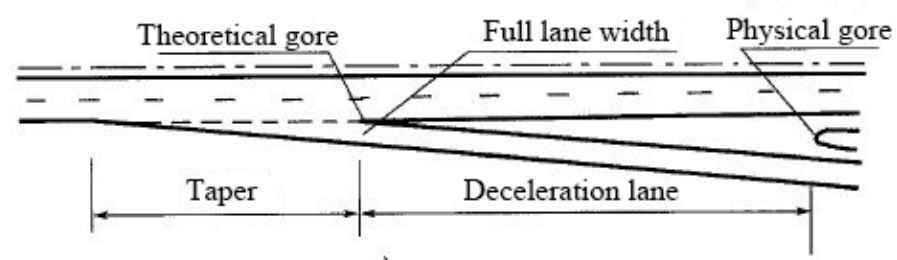

a)

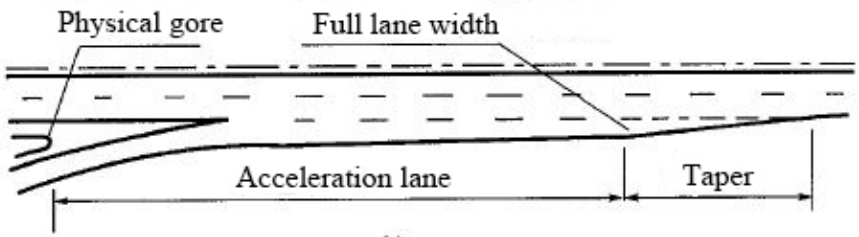

b)

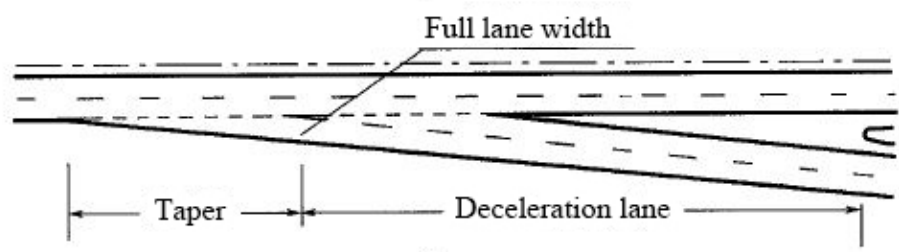

c)

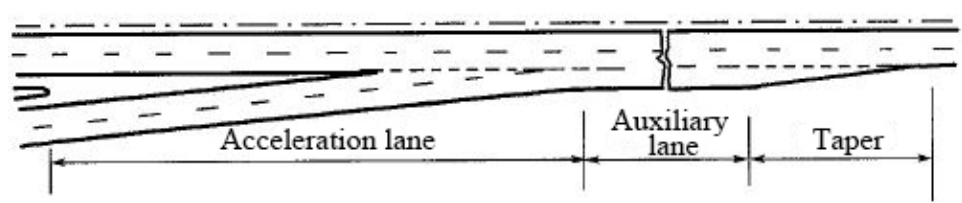

d)

Fig. 2 mode of speed-change lane

\section{Literature Review}

Richard A. Retting et al. ${ }^{[6]}$ conducted an experiment that pavement marking pattern was employed to narrowed the lane width of both the curve and a portion of the tangent section leading into the curve, and found it decrease the frequency of over speed in exit ramp successfully, which proved the effectiveness of pavement marking on driving behavior. The installation of the solid white line (prohibit merging line) in MUTCD ${ }^{[3]}$ is optional, which needs engineering study and judgment. Tien Dung Chu et al. ${ }^{[7]}$ studied the merging operation in Japanese urban expressway merging area, found the longer acceleration lanes did not always mean larger capacity: in the high density situation, the extension of acceleration lanes leaded to significant variations of merging positions. Thus, it is reasonable and of 
great significance to combine with principle of designing acceleration lane length in China, and study the layout of prohibit merging line for traffic safety and for reducing the variations of merging positions.

As regards to the characteristics of traffic stream in merging area in China, Xiaofang Yang ${ }^{[8]}$ used kinematic wave theory analyze the operation states in freeway merging area under different flow rate, and model was built to explain the evolvement rule of traffic flow. Tiezhu $\mathrm{Li}$ and Wenquan $\mathrm{Li}{ }^{[9,10]}$ investigate merging characteristics on freeway interchanges in many provinces and cities in China, found merging position often concentrate in the middle part of acceleration lane, and analyzed that merging in the middle part often went through acceleration, waiting and merging, it was unhurried, and could be thought safe and comfortable. In a certain degree, it underscored the need for prohibit merging line.

As regards to principle of designing acceleration lane length, considering the high occupancy of large trucks in China, commonly it was separated to acceleration segment, waiting segment, taper and calculated respectively ${ }^{[11-16]}$,namely vehicles accelerated to the merging speed, and then waited under this speed until the acceptable gap appeared, then finished the merging. Among that, the calculation of waiting segment was mainly using gap acceptance theory, the method was mature and lots of achievements have been obtained, which offset the deficiency of Chinese standard on designing acceleration lane length. Thus, this theory was also used in this paper to study the length design of prohibit merging line.

\section{Length design method of prohibit merging line}

The process of ramp vehicles speed on the acceleration lane and merge to mainline is affected by traffic volume on mainline. As the time distribution characteristics of traffic stream, the demand for prohibit merging varies with different traffic density and operation. With reasonable design of freeway, high density traffic should not appeared expect for traffic accidents occurred or other situation, and in high density traffic state ramp vehicles even need for deceleration due to the low speed of mainline vehicles ${ }^{[17]}$, the prematurely merging problem is not exist anymore, neither is the need for prohibit merging line, while this state is identified by that ramp vehicles' speed is faster than the adjacent mainline vehicles'. Therefore, in this paper only two states, low and middle densities of traffic were given qualitative analysis.

Mainline traffic in low density state. In this situation, ramp vehicles often accelerate and merge immediately without waiting, thus minimum length needed for acceleration is:

$$
\mathrm{L}_{0}=\frac{V_{f}^{2}-V_{r}^{2}}{26 a}
$$

where: $L_{\mathrm{v}}$ is minimum length needed for acceleration when mainline traffic is in low density state, $\mathrm{m} ; V_{\mathrm{f}}$ is operation speed of mainline vehicles, $V_{\mathrm{r}}$ is operation speed of ramp vehicles, $\mathrm{km} / \mathrm{h} ; a$ is acceleration of ramp vehicles, often valued $0.8 \sim 1.2 \mathrm{~m} / \mathrm{s}^{2}$.

Thus in low traffic density state of mainline, the length of prohibit merging line $L_{\text {(1) }}=L_{0}$.

Mainline traffic in middle density state. In this situation, ramp vehicles often accelerate to the merging speed (often slower than mainline vehicles') and move under this speed waiting for acceptable gap of mainline vehicles to merge.

(1) Accelerating

When mainline traffic is in middle density state, the length of acceleration segment is :

$$
\mathrm{L}_{\mathrm{a}}=\frac{V_{\mathrm{m}}^{2}-V_{\mathrm{r}}^{2}}{26 a}
$$

where: $L_{a}$ is the length of acceleration segment when mainline traffic is in middle density state, $m ; V_{m}$ is merging speed, namely the speed waiting for acceptable gap of mainline vehicles, $\mathrm{km} / \mathrm{h}$.

(2) Waiting 
The gap of mainline vehicles could generally be described by using Erlang distribution, cumulative probability of which is:

$$
P(h \geq t)=\sum_{i=0}^{k-1}(\lambda t)^{i} \frac{e^{-\lambda t}}{i !}
$$

where: $\mathrm{h}$ is an variable described gap; $\mathrm{k}$ is order of Erlang distribution, $\lambda$ is a parameter. $\mathrm{k}$ could describe states between free-flow and traffic jam. Higher value of $\mathrm{k}$ means traffic stream is more crowed and maneuvering within it is more difficult, and thus be used as the rough reflection of non-randomness, which increase with $\mathrm{k}^{[18]}$. Literature [19] concluded that when the traffic volume on outside lane of mainline is less than $250 \mathrm{veh} / \mathrm{h}$, the gap of it can be described by negative exponential distribution (first-order Erlang distribution); when the traffic volume is between 250 and 500veh/h, it can be described by second-order Erlang distribution; when the traffic volume is between 500 and $750 \mathrm{veh} / \mathrm{h}$, it can be described by third-order Erlang distribution. The k-order Erlang distribution density function is :

$$
f_{k}(t)=\frac{\lambda(\lambda t)^{k-1}}{(k-1) !} e^{-\lambda t}, t>0
$$

distribution function is:

$$
\mathrm{F}_{k}(\mathrm{t})=\mathrm{P}(\mathrm{h}<\mathrm{t})=1-\sum_{i=0}^{k-1} e^{-\lambda t} \frac{(\lambda t)^{i}}{i !}
$$

The expectation and variance of $\mathrm{h}$ is:

$$
\begin{aligned}
& \mathrm{E}(\mathrm{h})=\frac{k}{\lambda} \\
& \mathrm{D}(\mathrm{h})=\frac{k}{\lambda^{2}}
\end{aligned}
$$

Make $\mathrm{m}=\mathbf{E}(\mathrm{h})$, for the relationship between traffic volume on outside lane of mainline $\mathrm{Q}(\mathrm{veh} / \mathrm{h} / \mathrm{lane})$ and $\mathrm{m}$ is $: \mathrm{Q}=\frac{3600}{m}$, in practical application:

$$
\lambda=\frac{q k}{3600}
$$

Research show that, the value of acceptable gap $t_{0}$ is influenced by the characteristic of drivers, geometric design of merging area and speed, and the distribution is high in intermediate and low at two ends, the $85 \%$ distribution value is about 5 seconds ${ }^{[20]}$, thus value $\mathrm{t}_{0}=5 \mathrm{~s}$. Combine with Eq. 5 , the appearance probability of acceptable gap of vehicles on outside lane of mainline $\mathrm{P}_{c}$ is:

$$
P_{c}=P\left(h \geq t_{0}\right)=\sum_{i=0}^{k-1}\left(\lambda t_{0}\right)^{i} \frac{i^{-\lambda t_{0}}}{i !}
$$

Assuming a vehicle didn't merge to mainline until the nth gap appeared, namely the gap whose $h \geq t_{0}$, and each gap was independent and distributed randomly, thus the number of gaps waited could be described as geometry distribution $\mathrm{X} \sim \mathrm{G}\left(\mathrm{P}_{\mathrm{c}}\right)$. The cumulative probability of waiting $\mathrm{n}$ gaps $\mathrm{P}(\mathrm{n})$ can be calculated in Eq.10.

$$
\mathrm{P}(\mathrm{n})=\sum_{i=1}^{n} P_{n}=\sum_{i=1}^{n} P_{o}\left(1-P_{o}\right)^{i-1}
$$


Therefore, if the value of $\mathrm{P}(\mathrm{n})$ is given , $\mathrm{n}$ can be determined by Eq.10, and the length of waiting segment can be calculated by using merging speed ${ }^{[11]}$.

$$
L_{w}=\frac{n \cdot V_{\mathrm{m}}}{Q} \times 1000
$$

where: $L_{w}$ is the length of waiting segment when mainline traffic is in middle density state, $\mathrm{m}$; $\mathrm{Q}$ is traffic volume on outside lane of mainline, veh/h/lane.

As what studied in this paper was the length design method of prohibit merging line, some merging without fully accelerated and radical dangerous merging should be prohibited. Reference from the posted minimum speed's determining: use $15 \%$ distribution value of operation speed to reduce queuing and jam, and improve traffic safety ${ }^{[18]}$. Thus $\mathbf{P}(\mathbf{n})$ was valued as $15 \%$, the cumulative probability curve of geometry distribution with different $\boldsymbol{P}_{\sigma}$ was shown in Fig.3, in practical calculation, $\boldsymbol{P}_{\sigma}$ 's cumulative probability curve could be plotted by Excel, and when $\mathrm{P}(\mathrm{n})$ was $15 \%$, linear interpolation was used to determine the value of $\mathrm{n}$ (usually in the form of decimal).

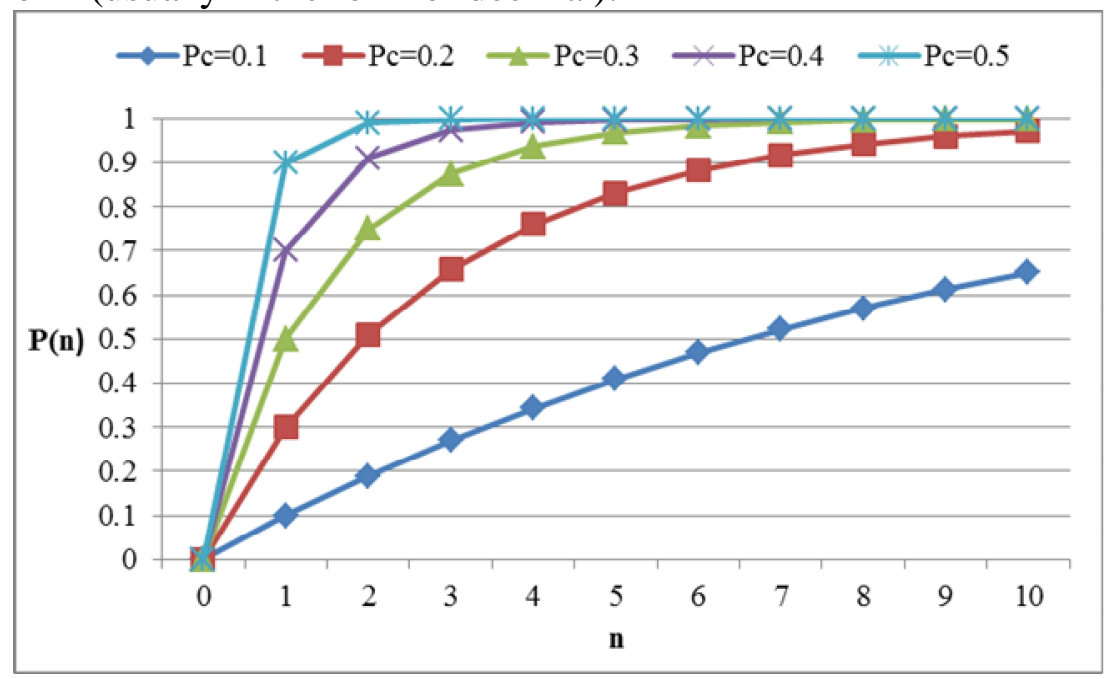

Fig. 3 cumulative probability curve of geometry distribution

Therefore, the length of waiting segment plus the length of acceleration segment equal to the length of prohibit merging line in middle traffic density state of mainline: $L_{(2)}=L_{a}+L_{w}$.

Comprehensively considering low and middle traffic density states, for encroachment to the reasonable merging position should be avoid while reducing the premature merging maneuver, the intersection of two states should be taken, namely $\operatorname{Min}\left\{L_{(1)}, L_{62} ;\right\}$ to be the design length of prohibit merging line. If the length of chevron markings in neutral area from physical gore to theoretical gore is less thanMin $\left\{\mathrm{L}_{(1)}, \mathrm{L}_{(2)}\right\}$, then prohibit merging line should be installed from the theoretical gore along downstream to offset the shortage. Notice that, to the tapered acceleration lane with double lanes and an auxiliary lane, the measurement of length of chevron markings in neutral area should be from physical gore along the ramp alignment downstream.

\section{Value of key variable}

Merging speed. Merging speed is suggested to be measured in field survey. If there is no measurement data, reference to the findings achieved by measurement in freeway merging area of many European countries: merging speed was averagely $20 \mathrm{~km} / \mathrm{h}$ slower than speed of mainline vehicles. For the consideration of safety, value the difference $15 \mathrm{~km} / \mathrm{h}^{[16]}$, thus

$$
V_{m}=V_{f}-15
$$

Traffic volume on outside lane of mainline. Traffic volume on outside lane of mainline Q (veh/h/lane) is also suggested to be measured in field survey. If there is no measurement data, reference to the following research: 
According to the field study, the relationship among traffic volume on outside lane of mainline Q, traffic volume of mainline $Q_{f}$ and traffic volume of ramp $Q_{r}$ was

$$
\mathrm{Q}=136+0.345 Q_{f}-0.115 Q_{r}^{[18]}
$$

\section{Demonstrating example}

The merging area in southwestern Maqun interchange hub in Nanjing, China was chosen as the example. Operation speed of mainline vehicles $V_{f}=50 \mathrm{~km} / \mathrm{h}$, operation speed of ramp vehicles $V_{\mathrm{r}}=$ $60 \mathrm{~km} / \mathrm{h}$, traffic volume of mainline was taken as the maximum service traffic volume in secondary service level $\mathrm{Q}_{\mathrm{f}}=1600 \mathrm{pcu} / \mathrm{h} / \mathrm{lane}^{[4]}$, traffic volume of ramp junctions with the main line was also taken as the maximum service traffic volume in secondary service level Q+Qr=1450 pcu/h ${ }^{[18]}$, according to Eq. 13, traffic volume on outside lane of mainline $\mathrm{Q}=589 \mathrm{pcu} / \mathrm{h}$, as referenced above, the gap could be described by third-order Erlang distribution. In current situation, the length from physical gore to theoretical gore is $42 \mathrm{~m}$, to the beginning point of taper is $167 \mathrm{~m}$, to the end of taper is $240 \mathrm{~m}$, the satellite imagery is shown in Fig.4.

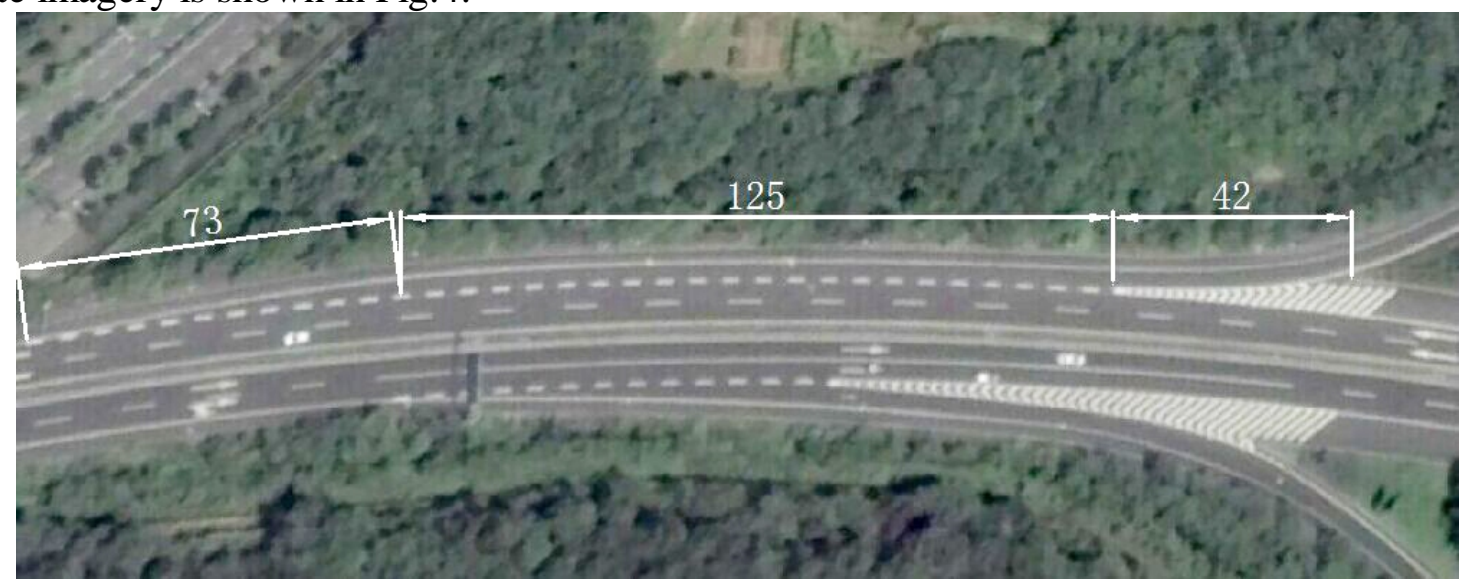

Fig.4 satellite imagery of the merging area in southwestern Maqun interchange hub

(1) Mainline traffic in low density state

According to Eq. 1 , take a $=1.2 \mathrm{~m} / \mathrm{s}^{2}, L_{0}=\frac{{Y_{f}^{2}}^{2}-V_{\mathrm{r}}^{2}}{26 a}=\frac{20^{2}-60^{2}}{26 \cdot 1.2}=90 \mathrm{~m}$;

Thus $L_{(1)}=L_{0}=90 \mathrm{~m}$

(2) Mainline traffic in middle density state

According to Eq. 2 and Eq.12, it can be drawn $V_{m}=65 \mathrm{~km} / \mathrm{h}, L_{a}=\frac{V_{m}^{n}-V_{i}^{n}}{26 a}=\frac{65^{2}-60^{2}}{26 \times 1.2}=20 \mathrm{~m}$;

According to Eq.8, $\lambda=\frac{Q \times 3}{3600}=\frac{599 \times 3}{3600}=0.49 s^{-1}$;

According to Eq.9,

$P_{o}=P\left(h \geq t_{0}\right)=e^{-\lambda t_{0}}\left(1+\lambda t_{0}+\frac{C \lambda t_{0} 2^{2}}{2}\right)=e^{-0.49 \cdot 5}\left(1+0.49 \cdot 5+\frac{(0.49 \cdot 5)^{2}}{2}\right)=0.56$

Excel was used to plot the cumulative probability curve when $P_{\varepsilon}=0.56$, and by using linear interpolation, $\mathrm{n}=0.27$.

According to Eq.11, $L_{w}=\frac{n \cdot v_{\mathrm{m}}}{q} \times 1000=\frac{0.27 \times 65}{5899} \times 1000=30 \mathrm{~m}$

Thus, $L_{\langle 2\rangle}=L_{a}+L_{w}=20+30=50 \mathrm{~m}$;

Comprehensively considering low and middle traffic density states, $\operatorname{Min}\left[L_{(1)}, L_{(2)}\right]=50 \mathrm{~m}$, for the length from physical gore to theoretical gore is only $42 \mathrm{~m}, 8$ meter length of solid white line (prohibit merging line) should be installed to offset, from theoretical gore extend along the road alignment downstream, while the width is relevant to design speed of the road according to the Chinese standard $^{[3]}$, here the width should be $15 \mathrm{~cm}$. 


\section{Conclusion}

In this paper, American standard MUTCD's provisions were referenced that solid white line (it's called prohibit merging line in this paper) could be installed from theoretical gore and extend along the road alignment downstream in merging area. A practical algorithm for designing length of the prohibit merging line with gap acceptance theory was proposed.

Notice that it is optional to install the solid white line in MUTCD, and need engineering study and judgment. However, in this paper only two states, low and middle density of mainline traffic, was considered. As analyzed above, for encroachment to the reasonable merging position should be avoided, the intersection of all states should be taken. In high density of mainline traffic, which is identified by that ramp vehicles' speed is faster than the adjacent mainline vehicles', traffic state ramp vehicles even need for deceleration due to the low speed of mainline vehicles, the prematurely merging problem is not exist anymore, neither is the need for prohibit merging line. Therefore, if high traffic density is in high frequency, there is no need for prohibit merging line.

From the designing principle of the prohibit merging line that reducing the premature merging maneuver without fully accelerated, it could be drawn that one of its warrant is the occurrence of a certain number of traffic accidents caused by that, while the specific indicators need further research. In addition, it is suggested that the values of variables are better obtained by field survey.

\section{Acknowledgements}

This work was financially supported by the Chinese "Twelfth five-year" National Sci-tech Support Plan, Key Technology and Equipment Research and Development for Improving Freeway Traffic Conditions (2014BAG01B01).

\section{References}

[1] Chinese Std. GB 5768-2009 (In Chinese).

[2] Chinese Std. JTG D82-2009 (In Chinese).

[3] American Std. MUTCD-2009.

[4] Chinese Std. JTG D20-2006 (In Chinese).

[5] Ming Li, Haizhong Zhang and Hao Gu: Journal of Chongqing Jiaotong University (Natural Science) Vol.24 (2005), p. 57-61 (in Chinese).

[6] R.A. Retting, H.W. McGee and C.M. Farmer C: Transp. Res. Rec. 1705 (2000), p. 116-121

[7] T.D. Chu, T. Miwa, and T. Morikawa: Procedia - Soc. Behav. Sci. Vol.138 (2014), p.105-115

[8] Xiaofang Yang, Qiang Fu and Hongjun Zhang: Journal of Tongji University (natural science) Vol.36 (2008), p.1652-1655 (in Chinese).

[9] Tiezhu Li, Wenquan Li and Ronggui Zhou: Journal of Highway and Transportation Research and Development Vol.18 (2001), p. 89-91 (in Chinese).

[10] Wenquan Li, Wei Wang and Tiezhu Li: Journal of Southeast University (Natural Science Edition) Vol.32 (2002), p.252-255 (in Chinese).

[11] Xiaofa Shi, Wenquan Li and Tiezhu Li: Journal of Henan University (Natural Science) Vol.30 (2000), p. 17-20 (in Chinese).

[12] Chune Wang: Journal of Highway and Transportation Research and Development Vol.30 (2013), p. 120-125 (in Chinese).

[13] Xiongjun He, Hui Liang and Jianping wang: Journal of Wuhan University of Technology (Transportation Science \& Engineering) Vol.29 (2005), p. 370-373 (in Chinese). 
[14] Jing Shi, Huina Shen: Journal of China \& Foreign Highway Vol.31 (2011), p. 309-314 (in Chinese).

[15] Zhongdan Yuan, Ling Xue and Weili Wang: Journal of Chongqing Jiaotong University (Natural Science) Vol. 28 (2009), p. 689-693 (in Chinese).

[16] Chun Zhao, Wei Deng and Ronggui Zhou: Journal of Highway and Transportation Research and Development Vol. 21 (2004), p. 103-107 (in Chinese).

[17] Huberman M: RTAC Forum Vol.4 (1982), p. 90-96

[18] Wei Wang, Xiucheng Guo: Traffic Engineering (Southeast University Press, China 2011) (in Chinese).

[19] Wenquan Li, Wei Wang and Ronggui Zhou: Journal of Highway and Transportation Research and Development Vol. 20 (2003), p. 114-117 (in Chinese).

[20] Xingjian Xue: Journal of Chongqing Jiaotong University (Natural Science) Vol.34 (2015), p. 95-99 (in Chinese). 